\title{
Intoxicação de anestésicos locais na prática odontológica: uma revisão da literatura
}

\author{
Intoxication of local anesthetics in dental practice: a literature review \\ Intoxicación por anestésicos locales en la práctica odontológica: revisión de la literatura
}

Recebido: 23/11/2021 | Revisado: 29/11/2021 | Aceito: 02/12/2021 | Publicado: 12/12/2021

Catarina Valéria Tavares De Oliveira Ferreira
ORCID: https://orcid.org/0000-0003-3667-0228
Centro Universitário Mário Pontes Jucá, Brasil
E-mail:catarina_oliveeira@hotmail.com
João Batista de Vasconcelos
ORCID: https://orcid.org/0000-0003-3213-5850
Centro Universitário Mário Pontes Jucá, Brasil
E-mail: jvasconcelos002@gmail.com
Ana Maria Guerra Costa
ORCID: https://orcid.org/0000-0002-6564-4718
Centro Universitário Mário Pontes Jucá, Brasil
E-mail: anaguerracosta@yahoo.com.br

\begin{abstract}
Resumo
A intoxicação sistêmica dos anestésicos locais (ALs) é uma das complicações mais temidas em anestesia pelo risco para o sistema cardiovascular, para o sistema nervoso central e, em particular, por desencadear parada cardiorrespiratória, mesmo em pacientes saudáveis. Os ALs interrompem a condução nervosa, interferindo na função de todos os órgãos nos quais ocorre a condução ou transmissão dos impulsos nervosos. Um dos principais fatores envolvidos é o rápido aumento da concentração plasmática. Os ALs junto aos vasos constritores reduzem o envenenamento por sua lenta absorção do sal anestésico. Além de fornecer anestesia e / ou analgesia para o paciente, o cirurgião-dentista que realizar o procedimento deve ter sempre em mente que a prevenção dessas complicações deve ser sempre observada. O objetivo deste artigo foi realizar um levantamento bibliográfico sobre a intoxicação por anestésicos locais, procurando enfatizar os principais sintomas, o tratamento e prevenção. Foram realizadas buscas bibliográficas nas bases de dados SciELO, MEDLINE e PubMed, com artigos publicados entre 2010 e 2021.
\end{abstract}

Palavras-chave: Anestesia; Anestésicos locais; Intoxicação.

\begin{abstract}
Systemic intoxication of local anesthetics (LAs) is one of the most feared complications in anesthesia due to the risk for the cardiovascular system, for the central nervous system and, in particular, for triggering cardiorespiratory arrest, even in healthy patients. ALs interrupt nerve conduction, interfering with the function of all organs in which the conduction or transmission of nerve impulses occurs. One of the main factors involved is the rapid increase in plasma concentration. ALs in the constrictor vessels reduce poisoning by their slow absorption of anesthetic salt. In addition to providing anesthesia and/or analgesia for the patient, the dentist performing the procedure must always keep in mind that the prevention of these complications must always be observed. The aim of this article was to carry out a bibliographical survey on intoxication by local anesthetics, trying to emphasize the main symptoms, treatment and prevention. Bibliographic searches were performed in the SciELO, MEDLINE and PubMed databases, with articles published between 2010 and 2021.
\end{abstract}

Keywords: Anesthesia; Local anesthetics; Intoxication.

\section{Resumen}

La intoxicación sistémica por anestésicos locales (AL) es una de las complicaciones más temidas en la anestesia por el riesgo para el sistema cardiovascular, para el sistema nervioso central y, en particular, para desencadenar parada cardiorrespiratoria, incluso en pacientes sanos. Los NA interrumpen la conducción nerviosa, interfiriendo con la función de todos los órganos en los que se produce la conducción o transmisión de los impulsos nerviosos. Uno de los principales factores implicados es el rápido aumento de la concentración plasmática. Los NA en los vasos constrictores reducen el envenenamiento por su lenta absorción de la sal anestésica. Además de proporcionar anestesia y / o analgesia al paciente, el odontólogo que realiza el procedimiento debe tener siempre presente que siempre debe observarse la prevención de estas complicaciones. El objetivo de este artículo fue realizar un relevamiento bibliográfico sobre intoxicación por anestésicos locales, tratando de enfatizar los principales síntomas, tratamiento y prevención. Se realizaron búsquedas bibliográficas en las bases de datos SciELO, MEDLINE y PubMed, con artículos publicados entre 2010 y 2021.

Palabras clave: Anestesia; Anestésicos locales; Intoxicación. 


\section{Introdução}

A intoxicação é um conjunto de efeitos nocivos representados por sinais e sintomas indicativos de um desequilíbrio orgânico, produzido pela interação nociva do agente químico com o sistema biológico (Santos, 2012), inibição da condução de nódulos sinoatriais e atrioventriculares, geradores cardíacos, arritmias, bradicardia e taquicardia, até fibrilação cardíaca (Barbosa, et al., 2018), distúrbios visuais, zumbido, contrações musculares, convulsões, inconsciência, coma, parada respiratória e depressão cardiovascular.

A toxicidade sistêmica de AL é uma complicação rara, mas potencialmente fatal, que pode ocorrer devido a altas concentrações plasmáticas do mesmo (Valencia, et al., 2013) sendo um dos principais fatores envolvidos na intoxicação e está diretamente relacionado a dose administrada, alterações na absorção local e sistêmica do anestésico, local de aplicação, distribuição tecidual, eliminação do fármaco, além de sua composição farmacológica estar associada ou não a agentes vasoativos (Barbosa, et al., 2010). Os ALs atuam no sistema nervoso deprimindo a atividade neuronal, mas seu mecanismo de ação farmacológico é distinto daquele dos anestésicos gerais. Portanto, intoxicações causadas por AL podem ser tão fatais quanto às decorrentes de anestesia geral, visto que doses extremamente altas dos mesmos podem deprimir a função respiratória, e podem levar à morte do paciente por asfixia (Santos, 2012).

O principal mecanismo de ação proposto para o AL está relacionado à ligação dessas substâncias aos receptores do canal de sódio $(\mathrm{Na}+)$, localizados na membrana dos nervos periféricos, o que leva à redução ou eliminação da permeabilidade destes íons,e, consequentemente, interrupção da condução nervosa (Pinheiro, et al., 2015). Como resultado, os ALs bloqueiam a condução nervosa, o que acaba interferindo na função de qualquer órgão ou tecido fornecido pela inervação bloqueado pela ação anestésica. Exercem uma ação inibitória sobre o sistema nervoso central (SNC) e periférico (SNP), deprimindo não apenas a geração e condução de impulsos nos nervos sensoriais como os nervos motores, mas também nos gânglios periféricos. As ações do AL causam o bloqueio da percepção sensorial, das funções neuromusculares e autonômicas locais (Santos, 2012).

Barbosa et al. (2018) mostraram que o AL leva à abolição das funções autonômicas e sensório-motoras, onde a alteração da condução nas fibras periféricas segue uma certa sequência como alteração da circulação sanguínea, daí uma depressão sensorial térmica, percepções dolorosas e táteis, depois aquelas relacionadas à pressão e vibrações e, finalmente, o sentido de propriocepção e controle motor. Esta sequência depende do diâmetro, porção e mielinização das fibras nervosas. A recuperação da função nervosa ocorre na ordem inversa. ALs são bases fracas, possuem baixa solubilidade em água e são instáveis à exposição ao ar, tendo pouco valor clínico nesta forma, portanto assume uma característica mais solúvel e estável em água. Entre os ALs comercializados, os mais amplamente utilizados em odontologia são lidocaína, prilocaína, mepivacaína, bupivacaína e articaína. As doses máximas recomendadas de AL são normalmente calculadas com base em miligramas de medicamentos por quilograma de peso corporal (Santos, 2012). Doses baixas ou altas de anestésicos podem afetar marcadamente a atividade do sistema nervoso e, assim, alterar funções orgânicas, incluindo funções respiratórias. Dentistas e outros profissionais de saúde devem escolher a solução anestésica dependendo da condição geral do paciente, levando em consideração peso, idade, estado de saúde e possíveis interações medicamentosas entre os ALs e os medicamentos usados pelo paciente.

O melhor tratamento das complicações sistêmicas decorrentes da anestesia local, sem dúvidas é a prevenção, por meio do contato verbal com o paciente, de qualquer sinal ou sintoma precoce de intoxicação, bem como do exame físico, escolhendo o sal anestésico mais seguro para cada caso específico, seguindo as dosagens recomendadas (Carvalho, et al., 2010).

\section{Revisão da Literatura}

Barbosa et al. (2018) revelaram que o primeiro anestésico local descrito foi a cocaína, extraída das folhas de Erythroxylon coca, em 1860, por Nieman, na Alemanha. Moreno e Maiz foram os primeiros a descrever o potencial do uso da cocaína como anestésico local em 1868, mas foi somente em 1884 que Koller a usou pela primeira vez para anestesia tópica do 
olho. A identificação da cocaína como derivado do ácido benzóico permitiu a síntese da benzocaína, um éster desse ácido, em 1890, por Ritsert. Em 1905, Einhorn e Braun sintetizaram procaína, derivada de ácido paraminobenzóico (PABA), mais solúvel em água e menos tóxico que benzocaína, compatível com uso sistêmico. Em 1943, Löfgren sintetizou lidocaína, derivada do ácido dietilaminoacético, onde se deu início a Era da Amida do tipo AL, relativamente livre de reações alérgicas, portanto comum com derivados do ácido paraminobenzóico. Franco et al. (2011) descobriram que os ALs bloqueiam a sensação de dor interferindo na propagação do impulso nervoso periférico.

A produção e a condução dos potenciais de ação são inibidas. Dados eletrofisiológicos indicam que os ALs não alteram significativamente o potencial normal de repouso da membrana nervosa, mas reduzem algumas respostas dinâmicas à estimulação nervosa em três partes: um grupo amino, uma cadeia intermediária em um grupo aromático. A forma de classificação leva em consideração que tipo de composto faz parte da cadeia intermediária do fármaco, aminoésteres para ésteres ou aminoamidas para amidas. Uma porção intermediária fornece a separação espacial necessária entre as duas extremidades e a ligação química entre a cadeia intermediária e o grupo aromático (lipofílico). Araújo, et al., (2008) avaliaram as teorias propostas para explicar os mecanismos de ação dos ALs, que podem ser classificados em duas categorias: atribuem o efeito anestésico à ligação desses compostos à proteína do canal de sódio e os que consideram a interação do AL com componentes lipídicos da membrana (conhecida como "hipótese lipídica ") como determinante do fechamento de canais de sódio.

Franco, et al., (2011) estudaram que em 1970, Lofstrom relatou casos fatais de overdose de AL. Os casos envolvendo pequenas doses administradas durante o tratamento odontológico foram, no entanto, exceções. Em 1979, Albright demonstrou uma correlação entre toxicidade e longo tempo de ação e AL altamente solúvel em gordura (bupivacaína, etidocaína). De 49\% de casos fatais, 43\% envolveram a bupivacaína. Pouco tempo depois, a Food and Drug Administration (FDA) retirou 0,75\% da bupivacaína do uso e recomendou uma administração mais cuidadosa desse medicamento. Desde então, a mortalidade associada a esta droga diminuiu significativamente. Um dos principais mecanismos envolvidos na toxicidade dos ALs é o aumento da concentração plasmática desses medicamentos em um curto período de tempo e doses intercostais ou excessivas. A via de administração dos ALs deve levar em consideração as condições clínicas pré-existentes (como insuficiência hepática), condições fisiológicas (gravidez), idade, alterações no equilíbrio ácido básico, alterações no estado de hidratação, também podem predispor à intoxicação. Boni et al. (2010) avaliaram que o rápido aumento da concentração plasmática é um dos principais fatores envolvidos na intoxicação, distribuição tecidual, eliminação da droga e adição ou não de agentes vasoativos. Carvalho et al. (2010) apontaram que um sinal sistêmico clássico de toxicidade é dormência perioral, porém, se este for o efeito desejado do bloqueio anestésico, outros sinais devem ser reconhecidos. Dentre os demais sinais clínicos incluem taquicardia, hipertensão, sonolência, confusão, zumbido e sabor metálico. Os sinais progressivos incluem tremores, alucinações, hipotensão e bradicardia. Os sinais tardios incluem perda de consciência, convulsões, arritmias, parada respiratória e circulatória.

O estudo de Feitosa et al. avaliou como os dentistas selecionam e previnem complicações sistêmicas ao usar ALs. Este estudo mostrou que o erro mais comum do cirurgião-dentista é padronizar um único tipo de anestésico para todos os pacientes, independentemente da condição sistêmica do paciente.

\section{Resultados e Discussão}

Os anestésicos locais são bases fracas agindo no axônio, bloqueando reversivelmente a geração e condução dos impulsos nervosos e seu usoé realizadoem grande escala, tanto em clínicas odontológicas quantomédicas. ALs são substâncias químicas que, em contato com a fibra nervosa, interrompem todos os modos de fluxo nervoso. Quando aplicadas às terminações nervosas ou troncos nervosos conduzindo sensibilidade, bloqueiam transitoriamente a transmissão do potencial de ação em todas as direções. (Carvalho, et al., 2010). Bloqueiam a abertura de canais iônicos na membrana celular neuronal, impedindo a neurotransmissão do potencial de ação. No entanto, a ligação específica ocorre no ambiente intracelular, sendo necessário que o 
anestésico local em sua forma molecular ultrapasse a membrana plasmática e bloqueie os canais de sódio. É provável que haja um segundo mecanismo de ação do AL, que envolve a inativação dos canais de sódio pela incorporação de moléculas de AL na membrana plasmática (teoria da expansão da membrana plasmática) (Edgcombe, et al., 2015). O segundo mecanismo de ação seria gerado pela forma não ionizada da neurotransmissão do AL, que atua de fora para dentro. As fibras nervosas têm sensibilidades diferentes do AL, as fibras pequenas são mais sensíveis do que as grandes, e as fibras mielizadas são bloqueadas mais rapidamente do que as fibras não mielizadas com o mesmo diâmetro. O bloqueio das fibras nervosas ocorre gradualmente, começando com perda de sensibilidade à dor, temperatura, toque, propriocepção e, finalmente, perda do tônus musculoesquelético (Edgcombe, et al., 2015).

$\mathrm{Na}$ odontologia, é difícil conseguir o controle adequado da dor sem a inclusão de vasoconstritores nas soluções de anestésico local, sendo o principal benefício a absorção lenta do sal anestésico, que reduz sua toxicidade e aumenta a duração da anestesia, permite o uso de pequenas quantidades da solução e aumentam o efeito anestésico, além dos vasoconstritores causarem redução do fluxo sanguíneo tecidual e, portanto, auxiliam na ação depressiva do anestésico no sistema nervoso (Santos, 2012). Existem soluções de AL que contêm vasoconstritores de dois tipos: aminas simpatomiméticas ou felipressinas aminas simpatomiméticas, em sua estrutura química, podem ou não ter núcleo catecol, por isso são denominadas, respectivamente, catecolaminas ou não catecolaminas, as antigas são representadas por epinefrina, norepinefrina e corbadrina. E de aminas, temos fenilefrina.

Os nomes genéricos de epinefrina, norepinefrina e corbadrina são sinônimos para adrenalina, norepinefrina e levonordefrina, respectivamente.As aminas simpaticomiméticas atuam nos receptores adrenérgicos encontrados na maioria dos tecidos do corpo. Esses receptores são de dois tipos: alfa ( $\alpha$ ), com os subtipos $\alpha_{1}$ e $\alpha_{2}$, ou beta ( $\beta$ ), com os subtipos $\beta_{1}, \beta, \beta 3$. A ação vasoconstritora é exercida pela interação com os receptores $\alpha 1$ (Andrade, et al., 2014). Os vasoconstritores mais comumente usados em combinação com soluções de AL são epinefrina (epinefrina), norepinefrina (norepinefrina), levonordefrina, fenilefrina e felipressina. $\beta$. O efeito é maior. Sua ação é bastante expressiva no miocárdio. Carvalho et al., (2010) mostraram que a dose máxima de adrenalina por sessão em pacientes saudáveis é de 0,2 mg. Para pacientes cardíacos, a dose máxima por sessão é de 0,04 mg.Complicações graves ocorrem com doses acima de 0,5 mg, com doses acima de $4 \mathrm{mg}$ geralmente, são fatais.

Entre os ALs comercializados, os mais utilizados na odontologia são: lidocaína, prilocaína, mepivacaína, articaína e bupivacaína. A lidocaína é considerada o anestésico padrão em odontologia, com a qual todos os outros são comparados aos anestésicos. O primeiro anestésico do grupo amino a ser sintetizado, em 1943 por Nils Lofgren, e inicia sua ação por aproximadamente 2-3 minutos (Santos, 2012). A dose máxima de lidocaína a 2\% recomendada pelos fabricantes é $4,4 \mathrm{mg} / \mathrm{kg}$, sem exceder $300 \mathrm{mg}$ (Carvalho, et al., 2010). Todos os ALs atravessam a barreira placentária, porém o uso de lidocaína com vasoconstritor é recomendado em mulheres grávidas, porque é um dos menos tóxicos ALs, sendo apenas nocivos se administrados em quantidades excessivas (Carvalho, et al., 2010).

O anestésico mais seguro para uso em pacientes pediátricos é a lidocaína a 2\%, com adrenalina 1: 200.000 (Santos, 2012). A prilocaína é duas vezes mais tóxica que a lidocaína e tem início de ação tardio, aproximadamente 2 a 4 minutos. A dose máxima de prilocaína 3\% é $4 / 4 \mathrm{mg} / \mathrm{kg}$ sem exceder $300 \mathrm{mg}$. É metabolizado mais rápido do que a lidocaína, no fígado e nos pulmões (Paiva, et al., 2005). O metabolismo hepático da prilocaína leva à formação da ortotoluidina, uma amina aromática responsável pela oxidação da hemoglobina em metemoglobina. A prilocaína aumenta a concentração plasmática de metemoglobina, o que promove hipóxia tecidual e aumento da incidência de toxicidade anestésica, ocorre cianose grave que não responde ao oxigênio. Nesses casos, a terapia de escolha é a administração intravenosa de azul de metileno, na esperança de deslocar a ortotoluidina para a molécula de hemoglobina (Barbosa, et al., 2010). A mepivacaína é amplamente utilizada na área odontológica, sendo classificada como um anestésico de duração intermediária tem potência e o dobro da toxicidade da lidocaína, com início de ação em torno de 1 minuto e meio a 2 minutos.A concentração dentária efetiva é de $2 \%$ com vasoconstritor e 3\% 
sem vasoconstritor (Santos, 2012). A dose aceitável de mepivacaína na presença ou ausência de vasoconstritor é 4,4 $\mathrm{mg} / \mathrm{kg}$, com uma dose máxima de $300 \mathrm{mg}$ (Carvalho, et al., 2010). Uma de suas vantagens é que essa substância pode ter efeito anestésico prolongado em comparação com outros ALs sem o uso de vasoconstritor (Santos, 2012). A articaína4\% foiaprovadapara uso nos Estados Unidos e no Brasil em 2000. Inicia sua ação de 1 a 2 minutos e meio. Sua dose máxima recomendada é de 7,0 mg / kg, não excedendo $500 \mathrm{mg}$. A articaína é mais potente que a lidocaína e menos tóxica (toxicidade 1,5 em comparação com a lidocaína de 2) (Santos, 2012). Há incidência de parestesia, provavelmente devido à concentração de 4\%, maior que a de outros anestésicos disponíveis no Brasil (Andrade, et al., 2014).

A ideia principal e mais importante no envenenamento por AL é a prevenção, selecionar corretamente o agente anestésico de acordo com o procedimento, aspirar antes de injetar o anestésico evitando a introdução do mesmo, na grande circulação, injeções lentas, manter contato verbal com o paciente quanto a sinais ou sintomas de intoxicação e principalmente, conhecer adequadamente as técnicas disponíveis e suas variantes (Santos, 2012). Ao procurar sinais de envenenamento por AL, deve-se ter em mente o quadro neurológico inicial e prestar atenção a sinais / sintomas específicos, como alterações na percepção sensorial geral, como gosto (gosto metálico na boca), alterações visuais (diplopia) e audição (zumbido), prestaratenção também aos possíveis distúrbios autonômicos que podem ser causados por medo, ansiedade, dor e desconforto durante procedimentos odontológicos (Santos, 2012).

Caso haja suspeita de intoxicação por ALs, as medidas seguintes devem ser tomadas:

1) Interrompa a administração da droga;

2) Ofereça oxigênio a $100 \%$ por máscara: O2 aumenta o limiar convulsivo e previne hipoxemia, melhorando o prognóstico do quadro;

3) Coloque o paciente em decúbito dorsal horizontal ou leve Trendelenburg, a fim de melhorar as perfusões cardíaca e cerebral;

4) $\mathrm{O}$ tratamento segue os algoritmos $\mathrm{P}-\mathrm{C}-\mathrm{A}-\mathrm{B}-\mathrm{D}$ ( $\mathrm{P}=$ Paciente consciente posicionado confortavelmente. $\mathrm{C}=$ Circulação, verificar pulso. $A=$ Vias aéreas, extensão do pescoço e elevação do queixo. $B=$ Respiração, ver os movimentos do tórax, ouvir os sons respiratórios e sentir o ar exalado. $\mathrm{D}=$ tratamento definitivo);

5) Caso o paciente não tenha acesso venoso estabelecido, providencie um se for necessário;

6) Mantenha monitorização adequada de oxigenação (oximetria de pulso), ritmo e frequência cardíaca (eletrocardiografia contínua) e pressão arterial.

7) O controle farmacológico das convulsões pode ser obtido com benzodiazepínicos por via venosa, particularmente com o Diazepan (5 a $10 \mathrm{mg}$ ) ou com Midazolan (5 a 15mg). Lembre-se sempre que estas medicações podem contribuir também para que o paciente entre em parada respiratória. Assim, você deve estar preparado para ventilá-loartificialmente;

8) Após o fim da reação, certifique-se que o paciente será examinado por um médico ou por um membro da equipe hospitalar, para determinar possíveis causas da reação. A avaliação deve incluir exames de sangue e provas de função hepática e renal.

9) Não deixar o paciente sair sozinho do consultório. Deve-se obter um acompanhamento com um adulto, caso a hospitalização não seja necessária.

10) Determinar a causa da reação antes de prosseguir com o tratamento que exige mais Als. (Santos, 2012).

\section{Conclusão}

Com base no exposto, conclui-se que é importante que o cirurgião-dentista esteja ciente dos aspectos farmacológicos como os vasoconstritores que têm muitos benefícios para os ALs, suas principais indicações e contraindicações, bem como possíveis reações locais e sistêmicas decorrentes de seu uso, conhecimento sobre as propriedades dos ALs, sua toxicidade e sua 
dosagem máxima. Além de executar um histórico médico completo ao paciente que procura atendimento odontológico, incluindo fatores de risco como os relacionados à idade e às comorbidade. É fundamental que a técnica selecionada seja aplicada corretamente diminuindo os riscos no uso de anestésicos locais e oferecendo maior conforto ao paciente. $\mathrm{O}$ anestésico local mais amplamente utilizado em odontologia é o cloridrato de lidocaína devido à sua toxicidade mais baixa, sendo prejudicial apenas se administrado em quantidades excessivas. Deve-se prevenir a intoxicação quando da utilização dos anestésicos locais.

\section{Referências}

Barbosa, B. A., Oliveira, L. K. R., Soares, M. F. M., Silva, T. O. S. S., Veloso, V. L., \& Marques, R. B. (2012). Intoxicação com anestésicos locais: Revisão de Literatura. Revinter-revista intertox de toxicologia, risco ambiental e sociedade, 11(2), 5-12.

Barbosa, M. P. L., Boni, C. L. A., \& Andrade, F. C. J. (2010). Conduta na intoxicação por anestésicos locais. Revista medica de minas gerais. $20(1)$ 24-30.

Carvalho, R. W. F., Pereira, C. U., Anjos, E. D., Filho, J. R. L., \& Vasconcelos, B. C. E. (2010). Anestésicos locais: Como escolher e prevenir complicações sistêmicas. Revista portuguesa de estomatologia, medicina dentária e cirurgia maxilo facial, 51(2), 67-127.

Carvalho, B., Fritzen, E. L., \& Parodes, A. G. (2013). O emprego dos anestésicos locais em Odontologia: Revisão de Literatura. Revista Brasileira 178 de Odontologia. 70(2), 178-81.

Dillane, D. \& Finucane, B. T. (2010). Local anestheticsystemictoxicity La toxicitesyste mique desanesthe'siqueslocaux. Can J Anesth/J CanAnesth.

Edgcombe, H. \& Hocking, G. (2015). Farmacologia dos anestésicos locais: Tutorial de anestesia da semana. John Radcliffe Hospital.

El-Boghdadly, Y. K., Pawa, A., \& Chin, K. J. (2018). Local anestheticxsystemic: current perspectives. Dovcepress-local and regional anesthesia, $11.35-44$.

Franco, V. A. M. (2011). Toxicidade dos anestésicos locais. Universidade Braz Cubas. 18 de May.

Gonzalez, M. M., Timerman, S., \& Oliveria, G. (2013). I Diretriz de Ressuscitação Cardiopulmonar e Cuidados Cardiovasculares de Emergência da Sociedade Brasileira de Cardiologia. Arq. Bras. Cardiol. 101(2).

Goyal, R. \& Shukla, C. R. N. (2012). Local anestheticsystemictoxicity (LAST) e Shouldwenotbeconcerned? Medical Journalarmed forces índia. 68.

Linsey, E., Picard, J., \& Weinberg, G. L. (2014). Local anaestheticsystemictoxicity. Oxford university, 15(3), 136-142.

Neto, E. M. R., Marques, L. A. R. V., Lotif, M. A. L., Lobo, P. L. D., Martiniano, C. R. Q., \& Ferreira, M. A. D. (2014). Toxicidade de anestésicos locais na prática clínica. Revista eletrônica de farmácia, vol. XI, 47-59.

Oda, Y. (2019). Local anesthetic systemictoxicity: proposedmechanisms for lipidresuscitationandmethodsofprevention. Journal of anesthesia, 1-3.

Pinheiro, A. C., Marques, J. F., Vieira, M. S., \& Almeida; L. S. B. (2015). Conhecimento de cirurgiões-dentistas sobre sinais e sintomas de toxicidade sistêmica associados ao uso de soluções anestésicas locais. RGO- revista gaúcha de odontologia, 63, 41-46.

Santos, F. C. (2012). Intoxicação anestésica: Causa, efeito e tratamento. Universidade estadual de londrina, 1-27.

Sekimoto, K., Tobe, M., \& Saito, S. (2017). Localanesthetictoxicity: acuteandchronic management. Acute medicine e surgery, 4. 152

Teixeira, R. N. (2014). Anestesia Local sem Vasoconstritor versus com Vasoconstritor. Universidade Fernando Pessoa Faculdade de Ciências da Saúde. Porto.

Valencia, M. I. B. \& Silva, J. F. V. (2013). Protocolo y necesidad de kit para toxicidad sistêmica por anestésicos locales. Revista colombiana de anestesiologia, 41(4), 274-279.

Yaddanapudi, S. (2011). Preventionof local anesthetic systemictoxicity. Journal of Anaesthesiology Clinical Pharmacology. 